\title{
CLANS2-A Code for Calculation of Multipole Modes in Axisymmetric Cavities with Absorber Ferrites*
}

\author{
D.G. Myakishev, Budker INP, Novosibirsk, Russia 630090 \\ V.P. Yakovlev, Omega-P, Inc, 345 Whitney Avenue, New Haven, CT 06511
}

\section{Abstract}

The code CLANS2 is an improved version of SuperLANS code [1] for calculation of axisymmetric cavities. This code permits the evaluation of multipole modes in cavities with partially loss dielectric and ferrite fillings. The code solves a complex eigenmode problem. A self-consistent problem is solved for frequency-dependent dielectric permittivity and magnetic permeability.

\section{INTRODUCTION}

The cavities with high loss ferrite filling are used in many areas. Ferrite insertions are used, for example, in superconducting accelerating cavities for HOM damping [2] and in high power RF amplifiers for self-excitation damping (see, for example, [3]). Most RF cavities have rotational symmetry, with a small amount of weak distortion produced by coupling elements. So 2D codes continue to play an important role in practical calculations. A calculation of the monopole modes in the presence of high loss materials can be produced using codes, such as CFISH [4] and CLANS [5]. In the present paper we describe a new code, CLANS2, for multipole mode calculations in axisymmetric systems. The multipole mode calculation is necessary, for example, for analysis and optimisation of transverse impedance of accelerating cavities with ferrite HOM dampers in cyclic accelerators [2]. It may also be useful for design and optimisation of RF sources, where operating modes have azimuthal variations, i.e., gyrotrons, gyroklystron, etc.

\section{SOLUTION METHOD}

CLANS2 is based on the same method as SLANS2 [5]. Unlike the scalar problem for monopole modes, the problem of multipole mode calculation is a vector problem. For example, the magnetic field is described by wave equation:

$$
\operatorname{rot} \frac{1}{\varepsilon} \operatorname{rot} \vec{H}-\omega^{2} \varepsilon_{0} \mu_{0} \mu \cdot \vec{H}=0,
$$

where $\varepsilon$ and $\mu$ are complex permittivity and permeability. Eliminating azimuthal field component, we obtain a system of the two equations for $H_{z}$ and $H_{r}$, which has no spurious solutions [6,7]. The boundary conditions for magnetic field on a metallic surface have the following form [8]:

$$
\frac{\partial H_{\tau}}{\partial n}+K \cdot H_{\tau}=0, \quad H_{n}=0
$$

where $\mathrm{K}$ - is the curvature of surface in $(r, z)$ plane. Solving the eigenvector problem for radial and axial components of magnetic field, we can reconstruct the azimuthal component of magnetic field and all components of electric field using Maxwell equations. Notice that it is also possible to solve the eigenmode problem for the same components of electric field. In this case the field equations have the same form, except that the boundary conditions on a metallic surface are [8]:

$$
\frac{\partial E_{n}}{\partial n}+\left(K+\frac{n_{r}}{r}\right) E_{n}=0, \quad E_{\tau}=0
$$

where $n_{\mathrm{r}}$ is radial component of unit vector normal to surface in $(r, z)$ plane. The two-component field equation is solved using the finite element method. We use a scalar eight-node rectangular isoparametric elements. These curvilinear elements provide a second-order approximation, and permit the describing of the geometry with high accuracy. The algebraic system of equations for the mesh node field values is obtained using Galerkin's method. The matrix system in our case is complex and asymmetric. To satisfy the boundary condition on a metallic surface we use the method described in [8]. We rewrite the equations for the mesh nodes on metallic surfaces for the normal and tangential field components: $H_{n}, H_{\tau}$ or $E_{n}, E_{\tau}$ and combine these equations to satisfy ( 2 or $2^{\prime}$ ). On ferrite or dielectric surfaces the problem of satisfying the boundary conditions is more complicated. In $(r, z)$ plane only the tangential field components $H_{\tau}$ and $E_{\tau}$ are continuous. So we rewrite the equations for $B_{n}, H_{\tau}$ or $D_{n}, E_{\tau}$ (instead of $H_{n}, H_{\tau}$ or $E_{n}, E_{\tau}$ ) for the mesh nodes on ferrite or dielectric boundary to satisfy the boundary conditions [5]. This method allows the use of a regular finite element mesh. To find several modes simultaneously in an arbitrary spectrum domain, we use subspace iteration method with frequency shift for an asymmetric algebraic problem [9]. If the permittivity and permeability of the lossy filling depend on frequency, the self-consistent problem is solved. The iterations are produced in the following way. The solution for fixed permittivity and permeability is used as an initial

\footnotetext{
* Work was supported in part by the grant JJ7100 from the International Science Foundation
} 
approximation. Then for this frequency the new values of permeability and permittivity are chosen. Using these values of permittivity and permeability, we find the new frequency by the method of inverse iteration with frequency shift, which provides the effective separation of required mode, and so on utill convergence. We use the frequency of the previous iteration as a new frequency shift. As a rule, several iterations are enough to obtain the self-consistent solution. Iterations are produced automatically if a file with permittivity and permeability frequency dependencies is prepared.

\section{TESTS AND EXAMPLES OF CALCULATIONS}

A spherical cavity with concentric spherical dielectric or ferrite insertion with high losses $(\operatorname{tg} \delta=1)$ was used as a test. For a mesh containing only 100 elements the difference between analytical calculations and CLANS2 results is less than $0.3 \%$ for the frequencies of the first 10 dipole modes. The code CLANS2 is currently used for multipole spectrum simulations in CESR superconducting cavity having ferrite HOM dampers [10]. The finite element mesh, which is used for dipole mode calculations, is shown in the Fig.1. The lines, where $r \cdot E_{\varphi}=$ const, are shown for two modes in the Figs. 2-4. Second mode is a "ghost" mode, which is located near the ferrite insertion and has very low quality factor. The field map $r \cdot E_{\varphi}=$ const for multipole modes is not, of course, a physical vector field, as for monopole modes, but it is helpful for mode identification. The code calculates the transverse impedance for modes, which is used for calculation of the beam instability growth rates. Another example of the multipole calculation is a variant of the room-temperature "single mode" cavity, proposed by V.M. Petrov [11]. A coaxial line with absorber is used to damp HOMs, both longitudinal and transverse. The position and size of the coaxial line is chosen in such a way that the fundamental mode does not propagate into the coaxial line. The perfectly matched load is simulated by the lossy ferrite filling of the end part of the coaxial line (see Fig. 6 and 7). The filling parameters are chosen in such a way as to eliminate reflection. The cavity spectrum and transverse impedance for multipole modes was calculated by CLANS2. The field map of the lowest dipole mode, which is damped by the coaxil load, is shown on Fig.6 and Fig.7.

\section{CONCLUSIONS}

A code has been developed that permits the deermination of multipole modes in cavities with partially loss dielectric and ferrite fillings. It may be used to solve the following problems:

- calculation of multipole modes in RF cavities with ferrite HOM dampers,
- $\quad$ simulation of the cavities of RF sources having lossy insertions,

- $\quad$ simulation of the RF cavities with a matched external load.

The code is written for Windows and UNIX platforms.

\section{ACKNOWLEDGMENTS}

The authors would like to thank Dr. B. Hafizi for careful reading of the manuscript.

\section{REFERENCES}

[1] D.G. Myakishev, V.P. Yakovlev, "An Interactive Code SuperLANS for Evaluation of RF-cavities and Acceleration Structures", in IEEE Particle Accelerator Conf. Rec.,1991, vol.-5, pp.3002-3004.

[2] S. Belomestnykh et al. Superconducting RF System for the CESR Luminosity Upgrade: Design, Status, And Plans. in Proc. of EPAC96 Conf., pp 2001-2003

[3] H.W. Matthews, W. Lawson, J.P. Calame, M.K.E. Flaherty, B. Hogan, J. Cheng, and P.E. Latham, "Experimental Studies of Stability and Amplification in a Two-Cavity Second Harmonic Gyroklystron", IEEE Trans. in Plasma Sci., v. 22, N5, pp. 825-833 (1994)

[4] M.S. de Jong et al., J. Microw. Power Electromagn. Energy, 27:136-142 (1992)

[5] D.G. Myakishev, V.P. Yakovlev, "The New Possibilities of SuperLANS Code for Evaluation of Axisymmetric Cavities", in Proc. of PAC95, pp 2348-2350

[6] M.M. Karliner, B.M. Fomel, V.P. Yakovlev, "LANS2 - A Code for Calculation of Oscillation with Azimuthal Field Variations in Axisymmetric Cavities." Preprint Budker INP 83-114 (in Russian).

[7] T. Weiland, "Transverse Beam Cavity Interaction, Part 2. Long Range Forces (On The Computation of Resonant Modes in Cylindrically Symmetric Cavities)," Preprint DESY 83-005,1983.

[8] Yu. Portugalov, "Method of Eigenmode Calculation in Cavities of Arbitrary Shape", Preprint IHEP 83-2, Serpukhov (in Russian).

[9] A.I. Fedoseev, "Subspace Iteration Method for Asymmetric General Eigenvalue Problems", Preprint IHEP 85-25, Serpukhov (in Russian).

[10] S. Belomestnykh, private communication.

[11] V.M. Petrov, V.N. Volkov, E. Kenjebulatov, private communication.

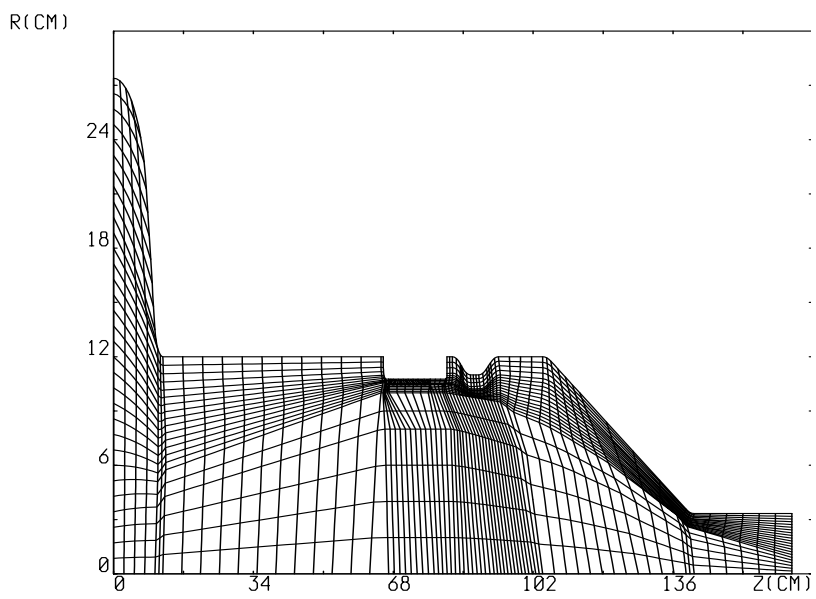

Figure 1: Finite element mesh for CESR superconducting cavity. 


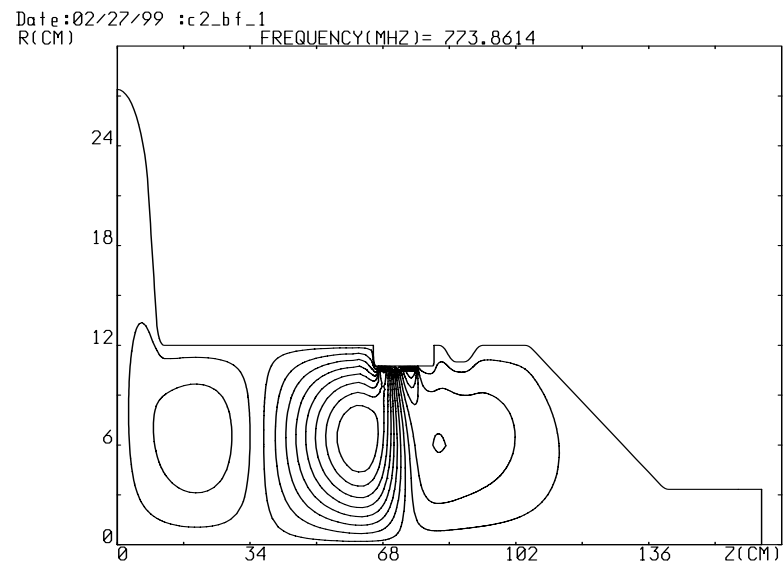

Figure 2: Field pattern of real part of a dipole mode of CESR superconducting cavity.

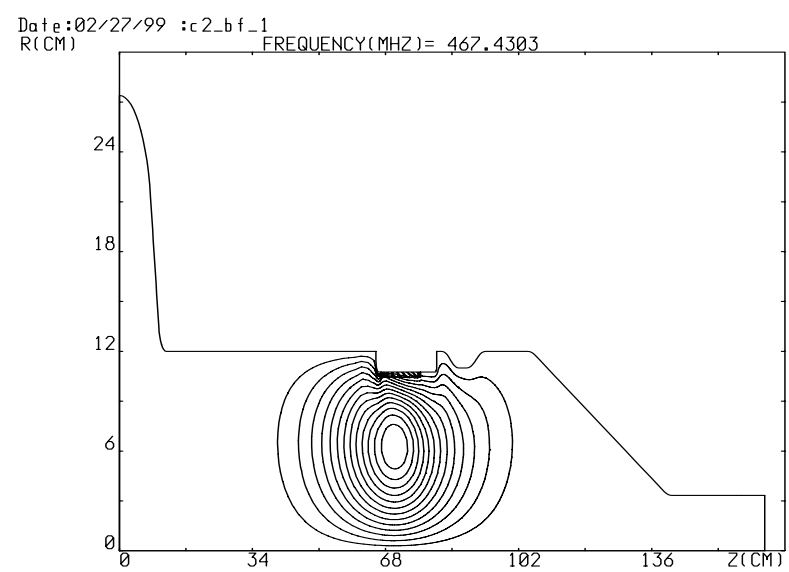

Figure 4: Field pattern of real part of a dipole "ghost" mode of CESR superconducting cavity.

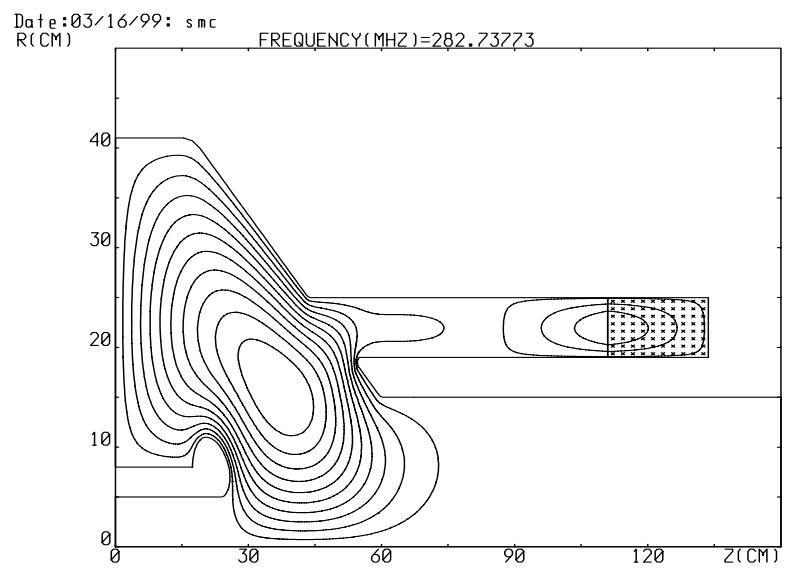

Figure 6: Field pattern of real part of a dipole mode of room-temperature "single mode" cavity.

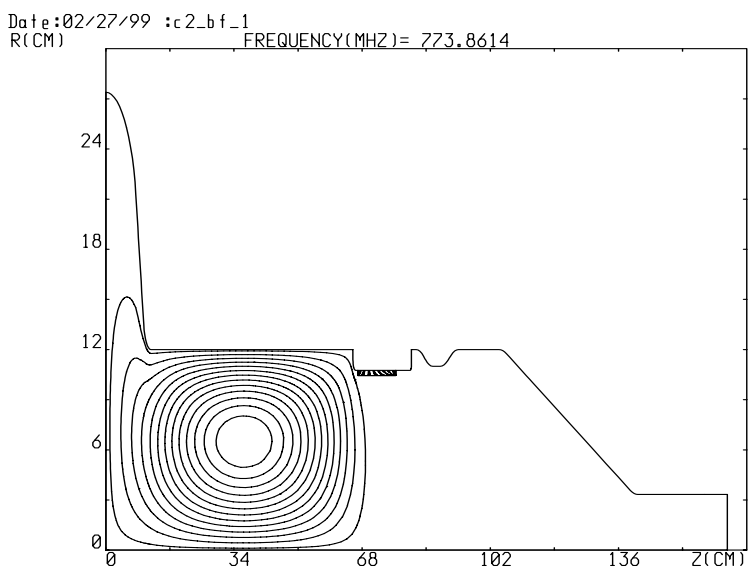

Figure 3: Field pattern of imaginary part of a dipole mode of CESR superconducting cavity

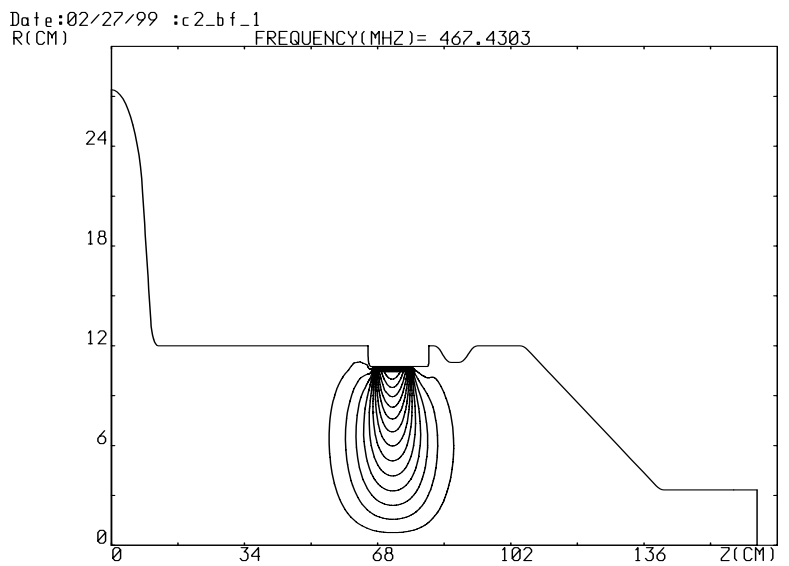

Figure 5: Field pattern of imaginary part of a dipole "ghost" mode of CESR superconducting cavity.

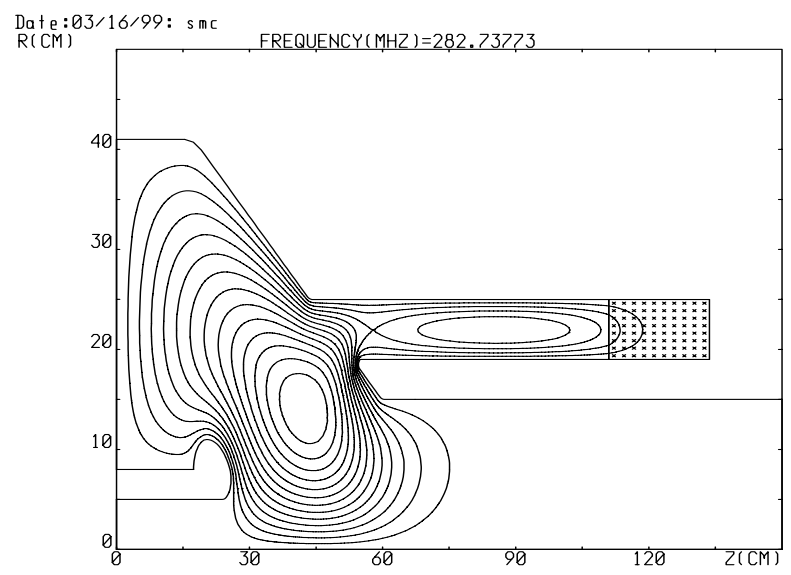

Figure 7: Field pattern of real part of a dipole mode of room-temperature "single mode" cavity. 